\title{
Multilineage Dysplasia Present
}

National Cancer Institute

\section{Source}

National Cancer Institute. Multilineage Dysplasia Present. NCI Thesaurus. Code C130048.

A hematologic test result indicating dysplastic changes in two or more of the myeloid cell lineages in the bone marrow. 